\title{
Belgeo
}

Revue belge de géographie

\section{Planifier avec le milieu : les liens complexes de l'analyse et de l'action}

Planning with the milieu: the complex connections of analysis and action

\section{Vincent Berdoulay}

\section{(2) OpenEdition}

1 Journals

Édition électronique

URL : http://journals.openedition.org/belgeo/16574

DOI : $10.4000 /$ belgeo. 16574

ISSN : 2294-9135

Éditeur :

National Committee of Geography of Belgium, Société Royale Belge de Géographie

\section{Édition imprimée}

Date de publication : 30 septembre 2003

Pagination : 231-254

ISSN : $1377-2368$

\section{Référence électronique}

Vincent Berdoulay, «Planifier avec le milieu : les liens complexes de l'analyse et de l'action », Belgeo [En ligne], 3 | 2003, mis en ligne le 30 octobre 2003, consulté le 05 février 2021. URL : http:// journals.openedition.org/belgeo/16574; DOI : https://doi.org/10.4000/belgeo.16574

Ce document a été généré automatiquement le 5 février 2021.

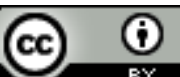

Belgeo est mis à disposition selon les termes de la licence Creative Commons Attribution 4.0 International. 


\title{
Planifier avec le milieu : les liens complexes de l'analyse et de l'action
}

Planning with the milieu: the complex connections of analysis and action

\author{
Vincent Berdoulay
}

1 L'évaluation de la capacité du milieu est un thème central dans l'histoire de la géographie, qui dépasse largement les aspects techniques de la discipline. L'enjeu est en effet épistémologique, car il conditionne le cadrage théorique et méthodologique de la question, et c'est sur ce point que j'insisterai dans cet article. Il est en effet possible d'avancer que les géographes ont depuis longtemps une capacité d'analyse et d'action qui a été sous-évaluée, non seulement par les autres spécialistes mais aussi par euxmêmes. Le regard rétrospectif qui est ici employé doit servir à mieux réfléchir sur la prospective de la discipline en rapport avec l'aménagement.

2 Aujourd'hui, malgré son apparence de radicale nouveauté, le souci de développement durable correspond à la recherche de principes d'action, dont bien des éléments philosophiques et conceptuels ont des racines anciennes. Les enjeux qu'il soulève sont multiples, quoique engageant toujours des paramètres environnementaux et territoriaux dont les prolongements se font sentir aux diverses échelles de l'aménagement (Vandermotten, 2002). Notamment à travers les notions de précaution, de participation, voire de résilience, la volonté de développement durable introduit dans l'élaboration des politiques publiques une sensibilité toute particulière pour la complexité. Le défi n'est alors pas tant de considérer celle-ci comme une contrainte, mais plutôt comme une opportunité d'action, comme une donnée avec laquelle concevoir l'action mais aussi la mener à bien.

3 C'est là que la notion de milieu reprend ses droits, car, plus que celle d'environnement, elle ouvre sur la complexité, sur le caractère hybride de ce qui est simultanément naturel et social. Le terme d'environnement a en effet été fortement connoté par des analyses cadrées par les sciences biophysiques et chimiques, ainsi que par les propositions d'action formulées par les ingénieurs qui s'en inspirent. En somme, c'est une notion qui renvoie à l'objectivation de la relation humaine à la nature et qui, par conséquent, en délaisse le pôle subjectif (Berdoulay, 2000). En revanche, le terme de 
milieu, par le flou même que cette vieille notion a entretenu, ne comporte pas ce type de parti pris. Il a l'avantage de désigner une relation qui peut être caractérisée autant par des données humaines que matérielles ou naturelles, quantitatives que qualitatives ; d'où le recours fréquent au mot milieu - et pas seulement en français - dès qu'il s'agit de capter la richesse et la complexité de cette relation (Buttimer, 1971; Berque, 1990, 2000). C'est pourquoi je privilégierai ici l'usage de ce terme, si pratique pour signifier mon acception très large, mais non prédéfinie, de l'ensemble complexe des relations que l'évaluation peut prendre en charge.

Mon propos est donc de présenter l'intérêt d'aborder le milieu, non comme une contrainte dont l'action, obéissant à d'autres logiques, doit se libérer, mais plutôt comme une opportunité d'action et un moyen pour la fonder. Il s'agit de réfléchir à la façon d'évaluer le milieu pour agir. Afin d'illustrer la démarche, j'utiliserai un exemple tiré de l'histoire de l'urbanisme, plus précisément au moment où celui-ci a cherché à fonder écologiquement l'aménagement des villes. Le cas de Barcelone servira de référence. Il me servira ensuite à dégager les grands défis épistémologiques et méthodologiques que la géographie doit relever pour aider à planifier avec le milieu, et non contre lui, et nous verrons qu'elle est mieux armée pour y arriver qu'on ne le pense, pour peu qu'elle ne tourne pas le dos à son passé.

\section{Des plans pour Barcelone, ou les différentes façons de penser le rôle du milieu}

5 Le cas de Barcelone est exemplaire car cette ville a fait l'objet depuis près de deux siècles de multiples propositions d'aménagement. Quoique la plupart n'ait pas abouti, plusieurs d'entre elles fournissent matière à réflexion car elles représentent des manières différentes d'aborder le milieu. En outre, elles sont souvent le fait de grands novateurs qui, en se penchant sur son cas, ont fait de Barcelone, à plusieurs reprises, un cas d'école. Et ce, au point où les plans d'urbanisme semblent avoir parfois obéi à une chronologie différente de celle rencontrée ailleurs.

6 Ainsi, une bonne partie de la ville est aménagée selon le plan préparé par Ildefons Cerdà et imposé en 1859 par le gouvernement central espagnol, malgré les desiderata de la bourgeoisie locale. On sait l'aspect révolutionnaire de ce plan, qui repose sur une théorie complète de l'urbanisation et qui préfigure bien des idées que l'urbanisme moderne mettra de l'avant à partir des années 1930 (Cerdà, 1867 ; Serratosa et al., 1994). De plus, on a souligné que l'innovation apportée par le plan Cerdà tient aussi à sa conception d'un réseau viaire qui, à partir d'une trame régulière d'avenues et de quelques transversales, doit maximiser les flux de toutes sortes, ne pas imposer une centralité particulière et assurer une ubiquité potentielle pour toutes les fonctions urbaines (Dupuy, 1991). Répondant au souci hygiéniste de l'époque, les voies sont larges, équipées d'égouts souterrains; et surtout, un espacement et une disposition en quinconces des immeubles est censée donner aux habitants un maximum d'aération, d'ensoleillement et d'accès à la verdure - projet qui sera complètement perverti au fil des ans en raison de la densification extrême des pâtés de maison. La démarche de Cerdà accorde donc une place à des données écologiques, mais seulement sous l'angle de l'hygiène. Pour le reste, le milieu n'intervient pas: la trame urbaine se déroule uniformément dans l'espace sans que l'on cherche à tirer parti des spécificités locales. L'idéal pour le projet est celui de faire table rase du milieu hérité du passé. Cela 
rappelle l'urbanisme moderne, où l'environnement n'est qu'une question de masses air, ensoleillement, zones vertes, etc. Le Corbusier dessina d'ailleurs à la fin des années vingt une esquisse de paysage barcelonais qui, à la manière de son Plan Voisin pour Paris, faisait table rase du tissu urbain existant au profit de gratte-ciel gigantesques espacés par de vastes zones vertes, avec, près du centre, le Montjuich qui devait être partiellement aplani pour faire place à un aéroport... Le Corbusier se plaisait à afficher, en la forçant jusqu'à la caricature, l'attitude de table rase vis-à-vis du milieu en place, pour la bonne raison que celui-ci était évalué principalement comme une contrainte.

7 L'inertie du plan Cerdà, les difficultés économiques et politiques, et le patrimoine architectural légué par l'art nouveau ont freiné l'ardeur des urbanistes modernes, dont les réalisations sont demeurées modestes en étendue, en même temps que l'immigration suscitait une expansion chaotique de la périphérie, surtout autour des années 1960 (Busquets, 1992). La reprise en main de l'urbanisme dans la période postfranquiste a permis la diffusion d'innovations à l'échelle de la ville, avec un rejet prononcé - quoique contesté en partie actuellement - des conceptions modernes, au profit d'une approche valorisant le projet et le cadre de vie des habitants (Bohigas, 1985 ; Berdoulay et Morales, 1999). Dans la recherche des précurseurs, les urbanistes barcelonais, comme ceux qui défendent ailleurs le projet urbain (Mangin et Panerai, 1999), se réclament des apports de l'urbanisme pré-moderne. Parmi eux, se note le nom de Léon Jaussely, qui précisément proposa un plan nouveau de Barcelone en 1905-1907. Or cet urbaniste fut un des leaders d'une véritable école de pensée urbanistique en France au début du XXe siècle, école qui cherchait à tirer parti de l'écologie urbaine et qui mérite d'être revisitée tant sa contribution a été occultée par les tenants de l'urbanisme moderne (Berdoulay et Claval, 2001). En tout état de cause, le plan selon Jaussely exprime de façon exemplaire comment l'évaluation du milieu peut, au contraire des urbanistes modernes, servir de fondement à la planification. Qui plus est, sa démarche s'inspire explicitement de l'apport des géographes vidaliens.

8 Pour mieux comprendre l'originalité de la position de Jaussely à l'égard du milieu, on peut la contraster brièvement avec un autre grand chef de file de cette école française d'écologie urbaine, Donat-Alfred Agache, connu pour ses plans tels que ceux de Dunkerque, Rio de Janeiro ou Curitiba. Très soucieux de bien évaluer la capacité du milieu - naturel, matériel et humain -, il s'efforçait d'en faire une étude détaillée, en prenant appui sur les méthodes d'enquête de terrain élaborées dans le cercle d'affinité des disciples de Le Play. La démarche d'Agache était de bâtir un diagnostic précis et objectif de la situation de la ville, en termes socio-économiques et de santé. Il devait lui permettre d'identifier les forces structurelles de la ville, mais aussi de repérer les dysfonctionnements de l'organisme urbain afin de proposer des remèdes visant à rétablir le bon fonctionnement de l'ensemble. En somme, son évaluation des capacités du milieu lui servait à renforcer les tendances lourdes qu'il y percevait. Sa prospective reposait donc sur la continuité des forces existantes, voire sur leur amplification. Tout autre était l'approche de Jaussely, et ce, dès son plan de Barcelone comme j'ai essayé de le montrer dans un livre récent dont je reprendrai ici une partie de l'argumentaire (Berdoulay et Soubeyran, 2002).

9 Jaussely répond en effet à un concours lancé en 1903 par la ville de Barcelone pour proposer un plan d'amélioration des liaisons entre les nouvelles parties de l'agglomération urbaine, suite à son expansion jusqu'à des localités qui se trouvaient au-delà du territoire couvert par le plan Cerdà. C'est bien ce que fait Jaussely dans 
l'avant-projet qu'il soumet et qui gagne le premier prix en 1905, le projet définitif étant accepté par la municipalité en 1907. Mais sa proposition va bien au-delà de la demande d'établir de nouvelles liaisons entre les parties d'une agglomération en expansion, et c'est précisément ce qui fera son succès, tant il révèle aux Barcelonais des moyens d'activer des possibilités latentes de leur milieu.

L'accueil des propositions de Jaussely connaît un succès considérable, ce qui fait de ce plan un véritable coup d'éclat du jeune architecte qui s'essaie comme quelques autres à renouveler l'art d'aménager les villes, ce qu'on désignera plus tard sous le nom d'urbanisme. Jaussely propose de ne pas limiter le plan au simple accompagnement en besoins techniques de communication et transport, comme l'exige effectivement la croissance urbaine, mais d'inscrire, et même réorienter, cette préoccupation dans un nouveau projet d'ensemble pour la ville et ses habitants. Et pour convaincre, Jaussely ne fait pas économie de procédés rhétoriques! Notamment dans son mémoire de 1907, il flatte le sentiment d'originalité et de fierté des Barcelonais, et il prend des accents qui sonnent agréablement aux oreilles des partisans de la ligue régionaliste catalane qui cherche les moyens d'accroître l'affirmation et le rayonnement de la région. Il aurait par ailleurs confié combien Barcelone l'avait surpris par son « mouvement», son " énergie vitale » et sa "position", voyant "que c'était la ville prédestinée à être la capitale de la Méditerranée et de la race limousine, provençale, catalane, etc. » (Gener, 1907, p. 58). Il joue aussi sur la communication picturale pour emporter l'adhésion. Les dessins qui accompagnent son mémoire frappent par leur côté visionnaire, exagéré, voire fantastique. Ils ne sont pas une illustration fidèle du plan; au contraire, ils sollicitent l'imagination pour entrevoir la direction vers laquelle on pourrait tendre. Il n'en reste pas moins que le plan de Jaussely se veut entièrement fondé sur une évaluation positive de ce que le milieu a à offrir. Car c'est d'elle que dépend sa démarche prospective.

11 Son évaluation de l'héritage du plan Cerdà s'inscrit dans cette démarche. Il en reconnaît quelques atouts, liés à la monumentalité, à l'hygiénisme, à l'équilibre entre le fermé et l'ouvert. En revanche, il lui reproche son manque de flexibilité vis-à-vis de besoins nouveaux, en même temps que la froideur et l'ennui que procure son uniformité. Surtout, il le juge en contradiction avec la culture locale, marquée par des coutumes où prime le goût pour la vie dans l'espace public, au rythme de promenades, fêtes et spectacles en plein air. La qualité de l'espace public doit être soignée, d'autant plus qu'il permet d'offrir à toutes les classes sociales la possibilité de s'exposer aux œuvres d'art qui y sont disposées à bon escient. L'aménagement urbain peut et doit faciliter l'activité culturelle de tout un chacun. Au fond, on ne peut plus tirer parti du plan Cerdà, car il est trop en disjonction par rapport au milieu humain de Barcelone, celui qui existe déjà et dont il cherche à amplifier la dynamique pour développer la vie civique.

Or ce milieu compte aussi des composantes naturelles à exploiter positivement. Pour augmenter la qualité de l'espace public et du cadre de vie, Jaussely reprend des idées qui avaient cours à l'époque et qui portaient autant sur l'aménagement de parcs, de jardins, de voies bordées d'arbres, que sur la morphologie des rues à la manière de Camillo Sitte (1902). Mais il est clair à ce propos, pour Jaussely, que le milieu naturel offre des opportunités à saisir. L'orientation des rues ou des avenues doit tenir compte de l'ensoleillement et de la direction des vents dominants, la situation de la ville entre mer et montagne jouant un grand rôle dans les recommandations. Il est intéressant à ce 
propos de voir combien, chez Jaussely, les capacités du milieu naturel sont révélées par la culture. Ainsi la mer, qui sert à des fins portuaires et industrielles, constitue à l'époque un potentiel inexploité pour améliorer le cadre de vie et promouvoir d'autres activités économiques, et notamment la fonction de station d'hiver pour l'aristocratie européenne. Jaussely recommande que, grâce au relief ou à la pente, le tracé des rues favorise la vue sur la mer et l'ouverture à son influence climatique bienfaisante.

L'existence, dans la morphologie de la ville déjà construite, de la trame laissée par le plan Cerdà constitue une contrainte dont Jaussely tient compte, comme de tout autre élément du milieu. Il s'efforce de l'intégrer dans la logique de son propre plan, notamment en prolongeant certaines voies pour relier la trame ancienne avec les localités périphériques. Il le fait avec d'autant plus d'aisance qu'il tient à ce que son plan comporte aussi une bonne dose de grandes avenues rectilignes, afin d'affirmer la grandeur et le pouvoir de Barcelone. C'est dans le même esprit qu'il recommande la conservation de certains éléments du patrimoine historique, qui témoigne de l'histoire de la société et de l'art à Barcelone.

En somme, si Jaussely est attentif aux particularités du milieu, qu'elles soient physiques ou humaines, passées ou actuelles, ce n'est pas nécessairement pour se conformer aux tendances qu'il y observe. Au contraire même, il semble bien vouloir introduire une rupture dans l'évolution qui se ferait sans l'intervention de son plan. Plus que de continuité, sa prospective en est une de bifurcation. Ainsi, le milieu est mobilisé de différentes façons. Il peut offrir des tendances à amplifier, comme le goût pour la vie publique et artistique dans la population barcelonaise. Il peut aussi contenir des possibilités encore inexploitées, comme la mer qui permet de promouvoir une nouvelle fonction, celle de station hivernale. Enfin, et surtout, le contexte régional - passé, actuel et futur - confère à Barcelone l'opportunité, sinon la mission, de rompre avec la routine du moment pour devenir une capitale culturelle, une des premières villes d'Europe.

15 C'est sur ce dernier point qu'insiste Jaussely, qui en fait la clé de voûte de son plan. Sa volonté de rupture s'y manifeste clairement, et il la déclare dès le début de son mémoire, quand il affirme que tout plan d'extension doit être quelque chose d'autre qu'une continuation du précédent. Il a bien conscience que les conditions de milieu peuvent se retourner, tant la probabilité des tendances actuellement anticipées peut changer sous le coup d'événements fortuits ou d'inventions technologiques encore impossibles à imaginer. Mais son analyse des capacités du milieu, manifestes ou latentes, est accompagnée de recommandations d'aménagement pour les développer ou pour leur donner une existence concrète, stabilisatrice des nouvelles orientations choisies. Le milieu transformé sous l'impulsion de l'urbaniste doit libérer des énergies qui, en retour, conforteront ces choix. Jaussely recommande que son plan soit effectué par étapes sur plusieurs dizaines d'années, afin de faire le point régulièrement et d'évaluer les réajustements nécessaires. La liberté humaine les garantit, rendant possible une planification à long terme.

16 En somme, l'aménagement doit se fonder sur le milieu, mais pas être déterminé par lui. Quoique non prédictible dans son évolution, le milieu s'offre à l'analyse en même temps qu'il sert de moyen d'action. Pour l'appréhender ou l'utiliser, Jaussely fait varier constamment la focale, passant de la petite à la grande échelle et sélectionnant les éléments pertinents. Il généralisera sa démarche en insistant que tout plan doit reposer sur la constitution préalable d'un « dossier » réunissant les résultats d'enquêtes auprès 
de la population, les études analytiques et les diagnostics. S'il s'accorde comme urbaniste un rôle très important dans l'élaboration des propositions d'action, plus important que dans les pratiques courantes, il n'en reste pas moins qu'il cherche à les fonder dans certaines aspirations de la société locale et ne peut échapper à les soumettre au contrôle démocratique des municipalités en charge de faire les choix.

$\mathrm{Au}$ moment où il concevait son plan de Barcelone, Jaussely ne disposait pas de beaucoup de ressources scientifiques pour comprendre la ville et son milieu. Il va toutefois faire siennes, très rapidement, les contributions des géographes vidaliens à l'étude de l'urbanisation, qui commencent à paraitre au début des années 1910 (Blanchard, 1912; Levainville, 1913). Leur approche globale, leur insistance sur le milieu et sur les contingences historiques, en font de véritables précurseurs de la compréhension des villes. Jaussely ne s'y trompe pas, et il n'aura de cesse d'en souligner l'importance pour fonder le travail de prospective de l'urbaniste. Il n'hésitera pas à proclamer à propos de l'urbanisme: «les géographes sont les pères de cette science » (Jaussely, 1919b, p. 188).

Nous voilà revenus à la géographie en tant que discipline. Interrogeons-nous donc maintenant, en partant de ce que Jaussely trouvait chez les géographes, sur le type de défis épistémologiques et méthodologiques qui sont posés à la géographie contemporaine par une évaluation des capacités du milieu ouverte à l'action.

\section{De quelques grands défis à la géographie}

La démarche d'évaluation prospective des capacités du milieu, si bien suggérée par la pratique urbanistique de Jaussely, entre en conjonction avec la contribution des géographes vidaliens sur la ville. Alors très remarquée en France et internationalement, cette contribution a le mérite de fournir pour la première fois une compréhension à la fois globale et approfondie de la ville (Aurousseau, 1924). Membres éminents de l'école de géographie menée par Paul Vidal de la Blache, ces auteurs de géographie urbaine fondent leur démarche sur une approche du milieu et des relations que l'homme entretient avec lui, qui repose sur une conception épistémologique et méthodologique cohérente, rapidement célébrée, à juste titre, par d'autres spécialistes (Febvre, 1922). Caractérisée de possibiliste, cette démarche se déploie sous le sceau de la complexité des interdépendances, par l'intermédiaire de l'adoption du regard écologique sur le milieu, qui consiste à mettre en cohérence, par le repérage de leurs interactions, des éléments qui semblent autrement disparates. C'est une des choses que Jaussely apprécie chez les géographes vidaliens: «il faut un grand esprit coordonnateur que seuls les géographes sont en mesure de donner " (Jaussely, 1919a, p. 125). Mais à ce point de vue interactionniste, ils en ajoutent un autre, qui le précise et l'opérationnalise, et qui relève de ce qu'on appellerait aujourd'hui un constructivisme. Ces géographes considèrent en effet comme actifs les deux termes de l'interaction homme-milieu et, pour les saisir, ils se concentrent sur les résultats, c'est-à-dire sur des médiations telles que les paysages, les régions, les villes, les villages ou les genres de vie.

D'une part, le milieu apparait comme quelque chose de complexe, d'instable, de fragile, dont l'évolution est marquée par la contingence. D'autre part, l'émergence de nouvelles formes de médiation entre le milieu et l'homme dépend du caractère actif de ces deux entités. A cet égard, le possibilisme s'appuie sur le néo-kantisme et le néo-lamarckisme 
de l'époque (Berdoulay, 1995; Berdoulay et Soubeyran, 1991). L'un comme l'autre insistent sur ce caractère actif joué par les deux pôles. Surtout, ils avancent l'idée que l'initiative et l'effort peuvent conduire à l'émergence de ces nouvelles formes de médiation. La liberté humaine, qui justifie la part d'initiative que l'homme peut essayer de prendre vis-à-vis des conditions de milieu, peut ainsi être intégrée dans la démarche scientifique.

21 La compatibilité de l'offre du géographe possibiliste avec les attentes d'un urbaniste tel que Jaussely est patente. Sa volonté d'adopter un regard écologique pour comprendre la ville est tout à fait analogue. Résultat d'interactions entre le milieu et les habitants, la ville relève du même schéma possibiliste, à la fois interactionniste et constructiviste. Surtout, ces auteurs partagent l'attention particulière qu'ils donnent à la culture, car elle manifeste la capacité humaine à révéler des possibilités latentes du milieu. Excluant tout déterminisme univoque, le possibilisme apparait effectivement utile pour l'analyse géographique; et il en va de même pour penser la prospective.

Non seulement la démarche possibiliste valorise la notion de milieu comme outil d'étude scientifique, mais elle en fait aussi un moyen pour agir. Le fait qu'il soit marqué de contingence, qu'il soit difficilement prédictible, n'empêche pas d'y identifier ce qui permettra de fonder et d'appuyer l'action proposée. On voit, ici, combien le possibilisme permet d'allier sans se contredire une prise en compte scientifique des données du milieu avec une démarche prospective qui prêche la rupture vis-à-vis des tendances qui ont été repérées. Il est bien évident que cette liberté d'action est toute relative aux interactions évaluées scientifiquement. Le possibilisme sert ainsi à fonder une prospective de la bifurcation. Des géographes vidaliens adopteront d'ailleurs explicitement cette idée que le milieu doit à la fois faire l'objet d'une étude scientifique rigoureuse et détaillée, et servir à fonder un projet d'aménagement qui introduit une bifurcation par rapport à ce qui prolongerait la situation existante (Vallaux, 1919). Ce rôle central accordé au milieu, et donc à la géographie, a légitimé la contribution active des géographes vidaliens aux débuts de l'institutionnalisation de l'urbanisme en France, mais il a en même temps stimulé les rivalités avec d'autres disciplines qui, en fin de compte, réussirent à les éliminer - élimination qui fut aussi celle d'un urbanisme fondé sur l'écologie urbaine... (Berdoulay et Soubeyran, 2001).

Quoi qu'il en soit, la démarche possibiliste garde toute sa pertinence, tant pour cadrer les analyses que pour fonder l'action. Elle n'impose pas une catégorie fixe, rigide, des types d'éléments qui doivent entrer dans ce qui fait milieu. Certes, les géographes ont eu tendance à privilégier les éléments qui correspondaient à ses dimensions naturelles. Mais, comme l'ont illustré Jaussely et les vidaliens, il n'est pas nécessaire d'autant se restreindre dans son choix, car le milieu reste relatif à la question posée, aux populations concernées ou à l'action envisagée. C'est la question toujours actuelle de la définition de ce qui est pertinent (Schmitz, 2001). C'est par la contingence et l'émergence qui caractérisent le milieu que la prospective peut tirer parti du potentiel de liberté qu'autorise celui-ci.

On sait toutefois que le possibilisme est tombé dans l'oubli, voire le discrédit, tant il a été stéréotypé, généralement réduit à l'inverse du déterminisme de l'environnement sur les actions humaines. Il est vrai que le développement de sa portée épistémologique et théorique a été handicapé par les difficultés que ses initiateurs ont éprouvées pour l'opérationnaliser. C'est qu'il y a un siècle, les outils analytiques faisaient gravement défaut. Seules l'observation sur le terrain, la cartographie et le raisonnement 
permettaient d'avancer. Même des concepts comme l'écosystème n'ont été créés, puis opérationnalisés, que plus tard. En somme, c'est essentiellement le versant langage, c'est-à-dire concepts et méthodes, qui n'a pas pu être à la hauteur des positions théoriques qu'inspiraient le possibilisme et par conséquent une certaine approche de l'action aménagiste.

L'approche systémique, par la valorisation qu'elle permet de l'échelle macroscopique, de la complexité et de l'émergence, a ouvert des voies intéressantes (Berdoulay, 1988 ; Turco, 1988; Roux, 1999). Liées aux sciences dites dures, elles conduisent à des modélisations de systèmes complexes (Berdoulay et Phipps, 1985 ; Pumain et al., 1989; Phipps et Langlois, 1997). Ces recherches, toutefois, demeurent encore difficilement applicables pour opérationnaliser toutes les préoccupations que j'ai soulevées ici. Je me limiterai donc maintenant à souligner quelques orientations conceptuelles, qui ne sont pas contradictoires avec ces nouvelles méthodes mais dont la portée demeure plus vaste. Sans aucune prétention à l'exhaustivité, je les ai retenues parce qu'elles me paraissent particulièrement propices à guider nos recherches.

On sait que le mouvement dit humaniste en géographie cherche à revaloriser et à reformuler la question des relations homme-milieu. En insistant sur les aspects qualitatifs, existentiels et subjectifs de cette relation, il leur donne la profondeur humaine qui manquait généralement aux études antérieures (Buttimer, 1971; Tuan, 1977, 1996 ; Berque, 2000). Pour ce faire, il a cherché appui dans la phénoménologie ou directement dans la pensée de Heidegger (Entrikin, 1976). Mais il faut ici souligner que ce mouvement n'a pas nécessairement à se mouler dans ces philosophies, car il reprend bien des thèmes et des postures de recherche qui avaient été esquissés dans d'autres contextes philosophiques (Ley et Samuels, 1978). Surtout, il confère au milieu une épaisseur et une richesse enfin appréciées à leur juste valeur, que les chercheurs avaient toujours eu du mal à saisir, même si d'autres voies que l'application de méthodologies scientifiques avaient été explorées à plusieurs reprises (Hard, 1969; Laplace-Treyture, 1998 ; Berdoulay, 2000). La plupart des travaux liés à ce mouvement humaniste demeurent toutefois peu, ou pas du tout, tournés vers l'aménagement. Or le souci de l'action n'est pas contradictoire avec ces préoccupations humanistes pour un élargissement de l'approche géographique, pour peu que l'on accorde plus d'attention à ses aspects interactionnistes et constructivistes. Pourtant, l'association ancienne entre la notion de milieu et celle de potentiel de liberté des acteurs avait implicitement désigné l'importance de se pencher sur le contexte de l'action du sujet, que celui-ci soit individuel ou collectif.

27 En approfondissant la critique post-structuraliste de la géographie, c'est-à-dire en réévaluant les fondements épistémologiques de celle-ci, il s'agit bien actuellement de réintroduire le sujet au centre des préoccupations (Berdoulay et Entrikin, 1998; Berdoulay, 1999). La volonté d'en tenir compte en tant qu'être autonome et actif va plus loin que de faire le simple recueil de ses représentations de l'environnement. Il ne s'agit pas non plus de considérer le sujet simplement comme un individu qui utilise dans son comportement des logiques - qu'elles soient d'ordre économique, identitaire ou environnemental - qui lui sont extérieures et qu'il ne ferait que réaliser. Dans la pratique, le sujet doit composer avec les contradictions que ces logiques génèrent; il doit donc assumer les tensions qu'elles lui imposent et construire sa propre ligne de conduite. Les assumer exige un investissement accru du sujet, qui doit mettre en 
cohérence son monde et, par là même, construire et reconstruire son identité. Le milieu est contrainte, mais il est aussi moyen de l'affirmation de soi.

C'est pourquoi l'intérêt de la notion de lieu a été souligné à nouveau. Elle permet d'attirer l'attention, et de focaliser la démarche, sur les interactions entre le sujet et son milieu. En conséquence du processus de résolution des tensions, il existe en effet une dynamique de co-construction du sujet et du lieu, ce dernier fonctionnant à son tour comme milieu dans une relation qui se poursuit. Il devient ainsi difficile de dissocier l'étude du sujet, du lieu, du milieu. En aménagement et développement territorial, le thème de la refondation territoriale illustre bien cette problématique (Despin, 2003). Il correspond à la volonté du sujet de réinvestir l'espace local, déstructuré par des processus plus globaux, en lui insufflant un sens nouveau, en recomposant les territorialités. Les acteurs de cette refondation fonctionnent bien comme des sujets en quête d'autonomie et désireux de faire émerger les nouveaux lieux de leur existence. Ils contribuent à ces phénomènes émergents que, précisément, recherche la prospective de la bifurcation que nous avons évoquée ci-dessus.

En somme, plutôt que de subir le milieu, le sujet s'affirme en faisant advenir du lieu. Le sujet sélectionne les éléments et dessine les contours du milieu avec lequel il interagit. Plus spécifiquement, c'est aussi le rôle que prend l'aménageur, sauf qu'il doit veiller à établir la bonne distance critique entre sa démarche normative et ce qui fait pour la population la singularité du lieu (Berdoulay et Entrikin, 1993). En faisant varier la distance qu'il prend vis-à-vis de son milieu, le sujet établit l'environnement pertinent de son action. Ainsi, l'attention récente portée en géographie à un sujet actif, loin de dévaloriser la notion de milieu, contribue fortement à la revaloriser, notamment en ce qui concerne les moyens potentiels qu'il offre pour l'action.

\section{Conclusion}

Le regard largement rétrospectif que j'ai ici privilégié a cherché à montrer que la notion de milieu n'est pas périmée. En phase avec l'intérêt contemporain pour l'assise écologique de la société et pour le développement durable, cette notion présente de multiples avantages. Notamment, elle revient à penser la planification autrement que d'une façon frontale, c'est-à-dire qui consiste à faire table rase de l'existant; elle permet de tisser des liens étroits entre la recherche fondamentale et la recherche pour l'action; et elle induit un rapprochement de la géographie avec les développements épistémologiques récents en sciences de la nature et de la société.

31 La première partie du présent article a attiré l'attention sur le souci qu'ont eu certains urbanistes de penser écologiquement l'aménagement des villes. L'exemple de Barcelone a servi à montrer que la question du milieu et de son analyse géographique pouvait jouer un rôle essentiel dans la façon de concevoir la prospective et l'action. Le plan Jaussely en particulier montre que, plutôt que d'opposer le milieu - comme contrainte à l'action, il convient de voir comment il constitue le moyen même de l'action. Il est donc clair que l'on peut très différemment analyser et mobiliser les capacités du milieu. Ce qui m'a incité, dans une deuxième partie, plus épistémologique, à revenir sur ce grand moment que fut le possibilisme comme théorie générale de l'action géographique reposant sur une évaluation de la capacité du milieu. Même si, faute d'un langage scientifique approprié, le possibilisme s'est longtemps dégradé dans la façon de le concevoir, sa pertinence intellectuelle demeure. Peut-être encore plus que les 
théories actuelles de la complexité, la question du sujet permet d'envisager un renouvellement de perspective sur le milieu, notamment en soulignant ses liens avec l'action.

L'évaluation des capacités du milieu renvoie ainsi à une géographie qui devient proactive, à une géographie où se mêlent intimement analyse et action, et elle engage cette discipline dans la vie sociale et politique. Mais en contribuant à l'espace public, les géographes doivent essayer de mieux préciser les modalités et les procédures de leur participation. Et ce sera pour le mieux, car leur discipline retrouvera, par là, sa vocation de science morale et politique.

\section{BIBLIOGRAPHIE}

AUROUSSEAU M. (1924), « Recent contribution to urban geography: A review », Geographical review, 14, pp. 444-455.

BERDOULAY V. (1988), Des mots et des lieux, Paris, Ed. du CNRS.

BERDOULAY V. (1995), La formation de l'école française de géographie (1870-1914), Paris, Ed. du CTHS. BERDOULAY V. (1999), « Géographie culturelle et liberté », in PITTE J.-R. \& SANGUIN A.-L. (dir.), Géographie et liberté, Paris, L'Harmattan, pp. 567-573.

BERDOULAY V. (2000), « Le milieu, entre description et récit; De quelques difficultés d'une approche de la complexité », in BERDOULAY V. \& SOUBEYRANO. (dir.), Milieu, colonisation et développement durable, Paris, L'Harmattan, pp. 25-37.

BERDOULAY V. \& CLAVAL, P., dir. (2001), Aux débuts de l'urbanisme français, Paris, L'Harmattan.

BERDOULAY V. \& ENTRIKIN J.N. (1993), « Singularité des lieux et prospective », Espaces et sociétés, 74-75, pp. 189-202.

BERDOULAY V. \& ENTRIKIN J.N. (1998), « Lieu et sujet. Perspectives théoriques ». L'Espace géographique, 27, pp. 75-89.

BERDOULAY V. \& MORALES M. (1999), « Espace public et culture : stratégies barcelonaises », Géographie et cultures, 29, pp. 25-42.

BERDOULAY V. \& PHIPPS M., dir. (1985), Paysage et système, Ottawa, Presses de l'Université d'Ottawa.

BERDOULAY V. \& SOUBEYRAN O. (1991), « Lamarck, Darwin et Vidal : aux sources naturalistes de la géographie humaine », Annales de géographie, 561/562, pp. 617-634.

BERDOULAY V. \& SOUBEYRAN O. (2002), L'écologie urbaine et l'urbanisme, Paris, La Découverte.

BERQUE A. (1990), Médiance. De milieux en paysages, Montpellier, GIP RECLUS.

BERQUE A. (2000), Écoumène, Paris, Belin.

BLANCHARD R. (1912), Grenoble, étude de géographie urbaine, Paris, A. Colin.

BOHIGAS O. (1985), La reconstrucció de Barcelona, Barcelone, Edicions 62. 
BUSQUETS J. (1992), Barcelona, Madrid, MAPFRE.

BUTTIMER A. (1971), Society and milieu in tne French geographic tradition, Chicago, Association of American Geographers \& Rand McNally.

CERDÀ I. (1867), Teoría general de la urbanización, trad. partielle : Théorie générale de l'urbanisation, Paris, Seuil, 1977.

DESPIN L. (2003), La refondation territoriale : entre le monde et le lieu, Paris, L'Harmattan.

DUPUY G. (1991), L'urbanisme des réseaux, Paris, A. Colin.

ENTRIKIN J.N. (1976), « Contemporary humanism in geography », Annals of the Association of American geographers, 66, pp. 615-32.

FEBVRE L. (1922), La terre et l'évolution humaine (en collaboration avec L. Bataillon), Paris, La Renaissance du Livre.

GENER P. (1907), « El plan de Reforma y Enlaces de los pueblos agregados », Anuario estadístico de la ciudad de Barcelona, pp. 57-77.

HARD G. (1969), « Dunstige Klarheit. Zu Goethes Beschreibung der italienischen Landschaft », Die Erde, 100 (2-4), pp. 138-154.

JAUSSELY L. (1907), Memoria,Proyecto de enlaces de la zona de Ensanche de Barcelona y de los pueblos agregados, Barcelone.

JAUSSELY L. (1919a), « Les cités dévastées par la guerre. Etudes de reconstitution. I. Un concours de plan de ville. La reconstitution de Chauny et de sa région », La Vie urbaine, 1-2, pp. 109-144.

JAUSSELY L. (1919b), « Chronique de l’“Urbanisme” », La Vie urbaine, 1-2, pp. 181-202.

LAPLACE-TREYTURE D. (1998), «Écriture savante et relation au voyage », Finisterra, 65, pp. 75-82.

LEVAINVILLE J. (1913), Rouen. Etude d'une agglomération urbaine, Paris, A. Colin.

LEY D. \& SAMUELS M. (dir.) (1978), Humanistic geography. Prospects and problems, Chicago, Maaroufa Press.

MANGIN D. \& PANERAI P. (1999), Projet urbain, Paris, Ed. Parenthèses.

PHIPPS M. \& LANGLOIS A. (1997), Automates cellulaires. Application à la simulation urbaine, Paris, Hermès.

PUMAIN D., SANDERS L. \& SAINT-JULIEN T. (1989), Villes et auto-organisation, Paris, Economica. ROUX M. (1999), Géographie et complexité. Les espaces de la nostalgie, Paris, L'Harmattan.

SCHMITZ S. (2001), « La recherche de l'environnement pertinent, contribution à une géographie du sensible ", L'Espace géographique, 30, pp. 321-332.

SERRATOSA A. et al. (1994), Cerdà. Ciudad y territorio, Madrid, Electa.

SITTE C. (1902), L'art de bâtir les villes, Paris, L'Equerre Editeur et D. Vincent, éd. de 1980 (trad. de l'original de 1899).

TUAN Y.F. (1977), Space and place, Minneapolis, University of Minnesota Press.

TUAN Y.F. (1996), Cosmos and hearth, Minneapolis, University of Minnesota Press.

TURCO A. (1988), Verso una teoria geografica della complessità, Milan, Unicopli.

VALLAUX C. (1919), « Les cités dévastées par la guerre, I. Péronne », La Vie urbaine, 1-2, pp. 77-95. 
VANDERMOTTEN C. (dir.) (2002), Le développement durable des territoires, Bruxelles, Editions de l'Université de Bruxelles.

\section{RÉSUMÉS}

L'article présente l'intérêt d'aborder le milieu, non comme une contrainte dont l'action, obéissant à d'autres logiques, doit se libérer, mais plutôt comme une opportunité d'action et un moyen pour la fonder. L'exemple historique de Barcelone sert à dégager les grands défis épistémologiques et méthodologiques que la géographie doit relever pour aider à planifier avec le milieu, et non contre lui.

This article shows the interest of not approaching the milieu as a constraint on action, from which it should be freed because it is obeying other logics. Milieu should rather be viewed as an opportunity for action and as a means for giving foundations to it. The historical example of Barcelona is used for showing what are the main epistemological and methodological challenges that geography should meet for helping to plan with the milieu and not against it.

\section{INDEX}

Keywords : milieu, planning, place, possibilism, Jaussely, Barcelona

Mots-clés : milieu, planification, lieu, possibilisme, Jaussely, Barcelone

\section{AUTEUR}

\section{VINCENT BERDOULAY}

Directeur de l'UMR 5603 (CNRS \& UPPA), Université de Pau, vincent.berdoulay@univ-pau.fr 